\title{
Rare-Earth Doped Upconverting Nanophosphors for Light- Mediated Biocidal Surface Development
}

\author{
Chanchal Hazra \\ INRS-EMT, University of Quebec, Canada \\ E-mail: chanchalhazra007@gmail.com
}

https://doi.org/10.54162/SD01-25201/02

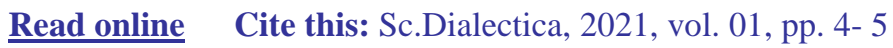

Keywords: Rare earth elements, Upconversion, Nanophosphors, Photocatalysis, Biocidal surface

\begin{abstract}
Since the earliest civilization, diminishing the occurrence and subsequent transmission of pathogenic microorganisms in the indoor environment has been one of the utmost priorities to the human society. In line with intensive research towards the surface disinfection through the use of several photocatalytic processes, rareearth doped upconverting nanophosphors (UCNPs) have recently drawn a great attention on the basis of their purely optical phenomenon of directly converting visible light into germicidal ultraviolet radiation (namely ultraviolet $\mathrm{C}$ ) via the unique photoluminescence process namely 'upconversion'. The efficient upconversion of abundant visible light into ultraviolet photons in the germicidal range and, consequently, effective biocidal action while coated onto surfaces enable UCNPs as a potential candidate to be used for inhibiting germ spreading through the inanimate surface in public places, hospitals and so forth.
\end{abstract}

\section{Discussion}

Beyond the boundary of either developing or developed country, it is desirable to build up a good quality indoor environment in the living society. To enrich this goal, it is essential to get rid of pathogenic microorganisms from the inanimate surface of the indoor environment through the use of sustainable and innovative disinfection technologies. During past decades, several techniques on surface disinfection have discussed the benefit of various photocatalytic processes. Photocatalysts (e.g. $\mathrm{TiO}_{2}$ ) have received widespread scientific and public interests as they use light energy to produce redox potential and germ-killing reactive oxygen species (ROS; hydroxyl radicals, superoxide radicals, etc.). ${ }^{[1]}$ Utilization of light energy in particular, ultraviolet (UV) radiation makes such technologies a more sustainable approach over the use of chemical disinfectants (e.g. alcohols, aldehydes, phenolics, etc.) or other energyintensive means (e.g. X- ray). Unfortunately, the existing technologies associated with UV light photocatalysts suffer from limitations such as low efficiency of visible light utilization. This fundamental feature restrict their scope to utilize visible light for antimicrobial application, i.e. converting low energy visible light which is again abundant in solar irradiation, and indoor lighting into higher energy ultraviolet $\mathrm{C}$ (UVC) radiation for killing germs from the inanimate surface of the indoor environment.

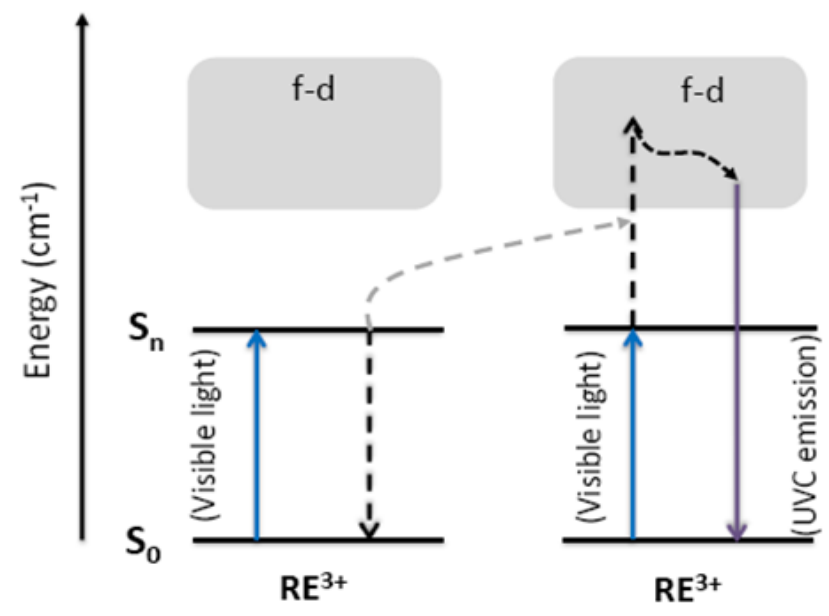

Figure 1. A simplified energy level diagram representing energy transfer upconversion (ETU) by $\mathrm{RE}^{3+}$ ions, where absorption of lower energy visible radiation results into the generation of higher energy UVC emissions. $\mathrm{S}_{0}$ denotes ground state and $\mathrm{Sn}$ denote excited state, where $\mathrm{n}$ refers to 1, 2. Solid lines represent absorbed or emitted photons, and dotted lines represent non-radiative transitions. Position of the $f-d$ band of $R E^{3+}$ ions solely depends on the nature of the host matrix.

To obtain such unconventional light conversion process, rare-earth $\left(\mathrm{RE}^{3+}\right)$ doped upconverting nanophosphors (UCNPs) play a crucial role as UCNPs itself could directly convert visible light into germicidal UVC radiation via the photoluminescence process of upconversion. ${ }^{[2]} \quad$ Typically, these $\mathrm{RE}^{3+}$ doped nanoparticles are composed of sensitizer(s) and 
activator(s) ions which are spatially distributed in an appropriate host matrix or in a dilute host-guest system. Successive absorption of one or more low energy photons and/or energy transfer (ET) generally promote the activators to the higher excited states and concurrently lead to the emission of higher energy radiation (one photon emission) spanning over a wide range, from the UVC to NIR spectral regions $^{[3,4]}$ (Figure 1).

$\mathrm{RE}^{3+}$ doped UCNPs can be achieved following different synthetic routes such as high temperature solid state method, sol-gel, microemulsion, co-precipitation, thermal decomposition, solvothermal, microwave irradiation, and simple colloidal reactions among others. Prior to employ in disinfection technology, it is essential to design sophisticated nanophosphors that would allow utmost upconversion efficiency and enable UVC emissions under low-power sunlight or ambient indoor light conditions. Such advances can be achieved through a proper choice of $\mathrm{RE}^{3+}$ content, precisely tailoring the shape and size of the nanophosphors, low vibrational host matrices, among others.

Thus, the UVC radiations originated from well-designed UCNPs play a pivotal role towards germ-killing activity from the inanimate surface of the indoor environment (Scheme 1). The UVC emissions are well known mutagenic agent that cause irreversible and heritable changes (mutations) in the

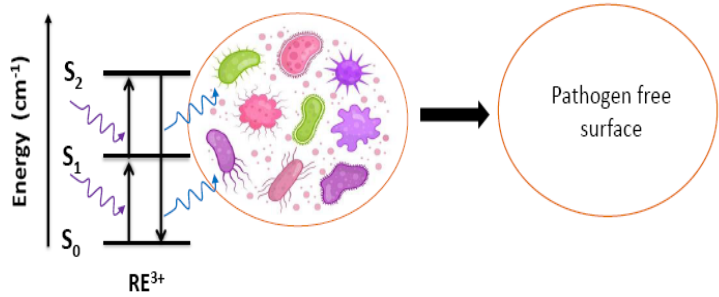

Conversion of visible radiation Inanimate surface with Pathogenic microorganisms to UV (UVB and UVC) emission Pathogenic microorganisms free inanimate surface

Scheme 1. Upconversion phosphor for light-activated antimicrobial activity. Simplified energy diagram represents upconversion (excited-state absorption, ESA) of visible light from $S_{0}$ (ground state) to $S_{1}$ and $S_{2}$ (excited state) to emit a UVC photons to killing pathogenic microorganisms from the inanimate surface.

cellular genetic material. Briefly, the UVC emission is pronouncedly absorbed by microbial, deoxyribonucleic acid (DNA) and ribonucleic acid (RNA) of the germs, causing covalent linkages between nucleic acid components to produce a variety of photoproducts that inhibit transcription. ${ }^{[5]}$

Based on this mechanism, upon visible light irradiation, the engineered nanophosphors while either coated onto surfaces as powders or fabricated in the form of ceramics, display self-sterilizing and anti-biofilm properties. Biocidal efficacy and biofilm inhibition are generally determined via kinetic viability assays and scanning confocal laser microscopy, respectively, using various test microorganisms. Thus, inanimate surfaces which can inherently remain pathogen free are a long sought-after tool for inhibiting pathogen transfer in hospitals and public areas. ${ }^{[2]}$

Overall, the present topic advances the scientific understanding of many aspects of upconversion materials that brings benefits to the field of applying upconversion phenomenon into sustainable cost-effective technology. Therefore, the future generation of our society should take more interest in the fundamental development of the current topic so that our society can have a better understanding in improving the quality of the indoor environment. Specially, after current Pandemic situation such an interdisciplinary learning has more relevance and promises towards monitoring healthy indoor environments.

\section{References}

[1] Y. Yan, C. Soraru, V. Keller, N. Keller and L. Ploux, "Antibacterial and Biofilm-Preventive Photocatalytic Activity and Mechanisms on P/F-Modified $\mathrm{TiO}_{2}$ Coatings", ACS Appl. Bio Mater., 2020, vol. 3, pp. 56875698 .

[2] S. L. Cates, E. L. Cates, M. Cho and J-H Kim, "Synthesis and Characterization of Visible-to-UVC Upconversion Antimicrobial Ceramics", Environ. Sci. Technol., 2014, vol. 48, pp. 2290-2297.

[3] S. Sarkar, B. Meesaragandla, C. Hazra and V. Mahalingam, "Sub-5 nm $\mathrm{Ln}^{3+-}$ doped $\mathrm{BaLuF}_{5}$ Nanocrystals: A Platform to Realize Upconversion via Interparticle Energy Transfer (IPET)", Adv. Mater., 2013, vol. 25 , pp. $856-860$.

[4] T. Cheng, R. Marin, A. Skripka and F. Vetrone, "Small and Bright Lithium-Based Upconverting Nanoparticles", $J$. Am. Chem. Soc., 2018, vol. 140, pp. 12890-12899.

[5] T. Dai, M. S. Vrahas, C. K. Murray, M. R. Hamblin, "Ultraviolet C irradiation: an alternative antimicrobial approach to localized infections?", Expert Rev Anti Infect Ther., 2012, vol. 10, pp. 185-195.

\section{Author's biography}

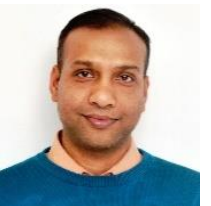

Dr. Chanchal Hazra is Postdoctoral Research Fellow in Chemistry at the National Institute of Scientific Research - Energy Materials and Telecommunication (INRS-EMT), University of Quebec, QC, Canada. He received his PhD in Chemical Sciences at the Indian Institute of Science Education and Research (IISER)-Kolkata, India in 2015. He worked as Postdoctoral Research Fellow at the Institute of Chemistry, State University of Sao Paulo (IQ-UNESP), SPBrazil. Dr. Hazra's research interest encompasses three distinct areas namely upconverting/downshifting nano/micromaterials based on rare earth (often called lanthanide) ions, novel photoactive materials and multifunctional nanoplatforms for applications in heavy metals/organic molecules sensing, environmental remediation as well potential applications in biomedical direction. 